\title{
Clinical impact of lenvatinib in patients with unresectable hepatocellular carcinoma who received sorafenib
}

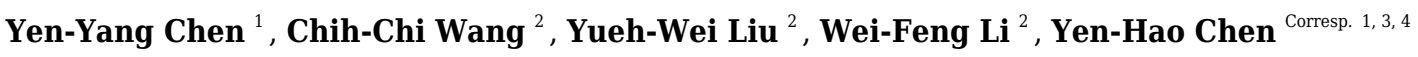 \\ ${ }^{1}$ Department of Hematology-Oncology, Kaohsiung Chang Gung Memorial Hospital and Chang Gung University College of Medicine, Kaohsiung, Taiwan \\ 2 Division of General Surgery, Department of Surgery, Kaohsiung Chang Gung Memorial Hospital and Chang Gung University College of Medicine, \\ Kaohsiung, Taiwan \\ 3 School of Medicine, Chung Shan Medical University, Taichung, Taiwan \\ 4 Department of Nursing, Meiho University, Pingtung, Taiwan \\ Corresponding Author: Yen-Hao Chen \\ Email address: alex2999@cgmh.org.tw
}

Background: Lenvatinib has been approved for use in the systemic treatment for unresectable hepatocellular carcinoma (HCC). This study aimed to investigate the efficacy and safety of lenvatinib in patients with unresectable HCC who received sorafenib.

Methods: A total of 40 patients who received lenvatinib after sorafenib were retrospectively identified: as second line in 20 patients, third line in 10 patients, and fourth line and later lines in 10 patients. The treatment response to lenvatinib was determined in accordance with the guidelines of the modified Response Evaluation Criteria in Solid Tumors (mRECIST) every 2-3 months after commencement of lenvatinib.

Results: Median progression-free survival (PFS) and median overall survival (OS) of the whole population were 3.3 and 9.8 months, respectively. The objective response rate was $27.5 \%$. Univariate and multivariate analyses showed that alpha-fetoprotein level $>400 \mathrm{ng} / \mathrm{mL}$ was an independent prognostic factor of worse PFS and OS. The clinical outcomes of lenvatinib therapy as second-line, third-line, or fourth line and later line treatment were similar, and previous response to sorafenib could predict the response to subsequent lenvatinib. Most adverse events were grades 1-2, and the majority of patients tolerated the side effects. Our study confirms the efficacy and safety of lenvatinib as second-line and later line treatment for patients with unresectable HCC who received sorafenib in clinical practice. 


\section{Carcinoma Who Received Sorafenib}

Yen-Yang Chen ${ }^{1}$, Chih-Chi Wang ${ }^{2}$, Yueh-Wei Liu²,

Wei-Feng $\mathrm{Li}^{2}$ and Yen-Hao Chen ${ }^{1,3,4^{*}}$

$6{ }^{1}$ Department of Hematology-Oncology, Kaohsiung Chang Gung Memorial Hospital and Chang

7 Gung University College of Medicine, Kaohsiung 833, Taiwan

8 2Division of General Surgery, Department of Surgery, Kaohsiung Chang Gung Memorial

9 Hospital and Chang Gung University College of Medicine, Kaohsiung 833, Taiwan

$10{ }^{3}$ Department of Nursing, Meiho University, Pingtung 912, Taiwan

$11{ }^{4}$ School of Medicine, Chung Shan Medical University, Taichung 402, Taiwan

13 Running title: Lenvatinib in $\mathrm{HCC}$ 
17 *Correspondence: Yen-Hao Chen, M.D., Ph.D.,

18 Department of Hematology-Oncology, Kaohsiung Chang Gung Memorial Hospital and Chang

19 Gung University College of Medicine, Kaohsiung, Taiwan

20 No.123, Dapi Rd., Niaosong Dist., Kaohsiung City 833, Taiwan (R.O.C.)

21 Tel: 886-7-7317123 ext. 8303; Fax: 886-7-7322402

22 E-mail: alex8701125@gmail.com

23

24

25

26

27

28

29

30

31

32 


\section{Abstract}

35 Background: Lenvatinib has been approved for use in the systemic treatment for unresectable

36 hepatocellular carcinoma (HCC). This study aimed to investigate the efficacy and safety of

37 lenvatinib in patients with unresectable $\mathrm{HCC}$ who received sorafenib.

38 Methods: A total of 40 patients who received lenvatinib after sorafenib were retrospectively

39 identified: as second line in 20 patients, third line in 10 patients, and fourth line and later lines in

4010 patients. The treatment response to lenvatinib was determined in accordance with the

41 guidelines of the modified Response Evaluation Criteria in Solid Tumors (mRECIST) every 2-3

42 months after commencement of lenvatinib.

43 Results: Median progression-free survival (PFS) and median overall survival (OS) of the whole

44 population were 3.3 and 9.8 months, respectively. The objective response rate was $27.5 \%$.

45 Univariate and multivariate analyses showed that alpha-fetoprotein level $>400 \mathrm{ng} / \mathrm{mL}$ was an

46 independent prognostic factor of worse PFS and OS. The clinical outcomes of lenvatinib therapy

47 as second-line, third-line, or fourth line and later line treatment were similar, and previous

48 response to sorafenib could predict the response to subsequent lenvatinib. Most adverse events 
49 were grades $1-2$, and the majority of patients tolerated the side effects. Our study confirms the

50 efficacy and safety of lenvatinib as second-line and later line treatment for patients with

51 unresectable HCC who received sorafenib in clinical practice.

52

53 Keywords: lenvatinib; hepatocellular carcinoma; sorafenib; survival

54

55

56

57

58

59

60

61

62

63

64 


\section{Introduction}

Hepatocellular carcinoma (HCC) is one of the most common aggressive malignancies

67 worldwide and is the second leading cause of cancer-related deaths in Taiwan. The well-known

68 risk factors for $\mathrm{HCC}$ include hepatitis B, hepatitis C, and alcoholic and nonalcoholic

69 steatohepatitis. Despite surveillance programs in high-risk patients, some patients with HCC

70 have advanced status when diagnosed, including multicentric intrahepatic spread, portal vein

71 thrombosis (PVT), huge tumor burden, and distant metastasis, contributing to poor prognosis

72 (Bertuccio et al. 2017; Bruix et al. 2016; Cabibbo et al. 2012).

73 Sorafenib is a small oral tyrosine and serine/threonine kinase inhibitor (TKI) that has been

74 proven as the first-line systemic treatment for unresectable HCC (Cheng et al. 2009; Llovet et al.

75 2008). Before 2018, sorafenib therapy is the only recommended strategy that can prolong overall

76 survival; apart from this, no other approved targeted therapy in the past 10 years has been

77 available as the first-line treatment for unresectable HCC (Cainap et al. 2015; Cheng et al. 2013;

78 Johnson et al. 2013). Recently, lenvatinib, a newly developed TKI targeting vascular endothelial,

79 fibroblast, and platelet-derived growth factor receptors, has become available as the first-line

80 treatment. The REFLECT trial showed that lenvatinib led to significantly better progression-free 
81 survival (PFS), time to progression (TTP), and objective response rate (ORR) than sorafenib and

82 was non-inferior to sorafenib in terms of overall survival (OS) (Kudo et al. 2018). Since then, an

83 increasing number of patients have been receiving lenvatinib therapy as the first-line treatment in

84 many countries, especially in Japan and Taiwan.

85 In the SELECT trial, a study that focused on the use of lenvatinib in radioiodine-refractory

86 thyroid cancer, there was no significant difference in survival between patients who received and

87 did not receive sorafenib (Schlumberger et al. 2015). This phenomenon was also observed in

88 patients with HCC. Recently, only few studies have showed no difference in survival outcomes

89 between patients who received targeted therapy and treatment-naïve patients (Hiraoka et al.

90 2019a; Hiraoka et al. 2019b; Hiraoka et al. 2019c). However, to the best of our knowledge,

91 information on the clinical impact of lenvatinib, such as prognostic factors, adverse events, and

92 correlation between sorafenib and lenvatinib response, in patients with HCC who received

93 sorafenib is limited in literature. The present study aimed to investigate the clinical impact and

94 safety of lenvatinib in patients with $\mathrm{HCC}$ who received sorafenib, including prognostic factors of

95 PFS and OS, survival comparison between different treatment line, presentation of side effects,

96 and correlation of response of lenvatinib and prior sorafenib. 
97

98

99

100

101

102

103

104

105

106

107

108

109

110

111 Materials \& Methods

112 Patient selection 
113 Between September 2018 and December 2019, the records of patients with unresectable

114 HCC who underwent treatment at the Kaohsiung Chang Gung Memorial Hospital were reviewed

115 retrospectively. First, only patients who received sorafenib followed by lenvatinib were enrolled.

116 Second, the included patients must have had at least one measurable target lesion for the

117 evaluation of treatment response. Third, the eligible patients had an Eastern Cooperative

118 Oncology Group Performance Status score of 0-1 and controlled hypertension. We excluded

119 patients with a history of second primary malignancy or concurrent hepatocholangiocarcinoma.

120 Finally, 40 patients who had available medical records and follow-up visits were identified.

123 The diagnosis of each patient with HCC was made according to pathology and dynamic

124 computed tomography (CT) or magnetic resonance imaging (MRI) of the liver in high risk

125 patients (HBV or HCV related liver cirrhosis) (Marrero et al. 2018; Omata et al. 2017). Staging

126 was determined according to the Barcelona Clinic Liver Cancer (BCLC) staging classification at

127 the time of lenvatinib treatment initiation (Llovet et al. 1999). Alpha-fetoprotein (AFP) was

128 measured before commencement of lenvatinib for each patient. 
131 Lenvatinib was orally administered at a dose of $10 \mathrm{mg}$ and $8 \mathrm{mg}$ once daily for patients with

132 body weight $\geq 60 \mathrm{~kg}$ and $<60 \mathrm{~kg}$, respectively. Adverse events (AEs) were evaluated according

133 to the National Cancer Institute Common Terminology Criteria for Adverse Events version 4.0,

134 and the worst grade for each AE was recorded. of the liver to be evaluated for treatment response to lenvatinib in accordance with the guidelines of the modified Response Evaluation Criteria in Solid Tumors (mRECIST) (Lencioni \& Llovet 2010) every 2-3 months after commencement of lenvatinib. Assessment was performed

141 independently by two radiologists blinded to any information about the patients'

142 clinicopathologic features or prognosis. 
145 Statistical analyses were performed using SPSS 19 software (IBM, Armonk, NY, USA).

146 Statistical analyses of group differences were performed using the Chi-square test with

147 Bonferroni correction for categorical variables. PFS was defined as the time from the initiation

148 of lenvatinib treatment to disease progression or death from any cause, and OS was calculated

149 from the date of lenvatinib treatment initiation to death or last living contact.

150 Kaplan-Meier method was used to estimate PFS and OS, and the log-rank test was

151 performed to evaluate the differences between groups for univariate analysis. The hazard ratio

152 (HR) with 95\% confidence interval (CI) and P-values were calculated to quantify the strength of

153 the associations between the prognostic parameters and survival. Cox proportional hazards

154 model was performed for multivariate analysis. All tests were two sided, and a P-value of less

155 than 0.05 was considered to indicate statistical significance.

Ethics statement

158

This retrospective study was approved by the Chang Gung Medical Foundation Institutional 
160 guidelines, and written informed consent was waived by the Chang Gung Medical Foundation

161 Institutional Review Board.

162

163

164

165

166

167

168

169

170

171

172

173 Results

174 Patient characteristics 
175 We identified 40 patients with unresectable $\mathrm{HCC}$ who received lenvatinib as second-line or

176 later line treatment at our institution between September 2018 and December 2019. There were

17735 men and 5 women with a mean age of 58 (range, 34-78) years. All patients had liver cirrhosis

178 in our study. The Child-Pugh classification was A in 31 (77.5\%) patients and B in $9(22.5 \%)$

179 patients, whereas the BCLC staging classification was B in $3(7.5 \%)$ patients and $\mathrm{C}$ in 37

180 (92.5\%) patients. Regarding viral hepatitis, 29 (72.5\%) patients had hepatitis B virus (HBV)

181 infection, 7 (17.5\%) patients had hepatitis C virus (HCV) infection, and $4(10.0 \%)$ were negative

182 for HBV or HCV. Nineteen (47.5\%) patients had macrovascular invasion, including 11 patients

183 (27.5\%) with main PVT. In addition, 24 (60.0\%) patients underwent hepatectomy before

184 lenvatinib treatment, and extrahepatic spread was noted in $32(80.0 \%)$ patients. There were 17

185 (42.5\%) patients with AFP $>400 \mathrm{ng} / \mathrm{mL}$. At the time of analysis, the median period of follow-up

186 was 15.1 months for the 15 survivors and 8.9 months for all 40 patients. The clinicopathologic

187 parameters of the patients are shown in Table 1.

188

189

Clinical outcomes of lenvatinib treatment 
191 univariate analysis showed no significant differences in all parameters except AFP $>400 \mathrm{ng} / \mathrm{mL}$.

192 The 17 patients with AFP $>400 \mathrm{ng} / \mathrm{mL}$ had worse PFS as compared to the 23 patients with AFP

$193<400 \mathrm{ng} / \mathrm{mL}(2.7$ months versus 4.2 months, $\mathrm{P}=0.020$, Figure $2 \mathrm{~A})$. Multivariate analysis

194 revealed that $\mathrm{AFP}<400 \mathrm{ng} / \mathrm{mL}(\mathrm{P}=0.024 ; \mathrm{HR}, 0.46 ; 95 \% \mathrm{CI}, 0.23-0.90)$ was an independent

195 prognostic factor for better PFS.

196 Regarding OS, the median OS of the whole population was 9.8 months (Figure 1B). No

197 significant differences were observed in terms of all parameters except AFP $>400 \mathrm{ng} / \mathrm{mL}$ in the

198 univariate analysis. As expected, a superior OS was found in 23 patients with AFP $<400 \mathrm{ng} / \mathrm{mL}$

199 when compared with the other 17 patients with AFP $>400 \mathrm{ng} / \mathrm{mL}$ (not reached versus 6.1

months, $\mathrm{P}<0.001$, Figure 2B). According to the multivariate analysis, AFP $<400 \mathrm{ng} / \mathrm{mL}(\mathrm{P}<$

201

0.001; HR, 0.19; 95\% CI, 0.08-0.46) was an independent prognostic factor for superior OS. The

results of univariate and multivariate analyses of PFS and OS in 40 patients with unresectable

HCC who received lenvatinib after failure of sorafenib treatment are shown in Tables 2 and 3.

204 
206 The response to lenvatinib treatment was determined according to the mRECIST criteria,

207 including $1(2.5 \%)$ patient with complete response (CR), $10(25.0 \%)$ with partial response (PR),

$20816(40.0 \%)$ with stable disease (SD), and $13(32.5 \%)$ with progressive disease (PD). The 6-

209 month PFS rates were $45.7 \%$ and $18.8 \%$ in the PR and SD groups, respectively; the 1-year OS

210 rates were $53.3 \%$ in the PR group, $55.6 \%$ in the SD group, and $8.5 \%$ in the PD group.

211 In our study, lenvatinib therapy was used as second-line treatment in $20(50.0 \%)$ patients,

212 third-line treatment in $10(25.0 \%)$ patients, and fourth-line and later lines in $10(25.0 \%)$ patients.

213 The median PFS and OS were 3.1 months and 8.1 months, respectively, for the second-line

214 treatment; 3.7 months and 11.5 months for the third-line treatment; and 3.1 months and 12.0

215 months for the fourth line and later line treatments. The results of the survival analyses of the

216 treatment effects of lenvatinib are shown in Table 4.

217 We also analyzed the correlation of treatment responses between sorafenib and lenvatinib.

218 There were 6 patients with PR to sorafenib previously, and they all achieved CR or PR with

219 lenvatinib treatment. Among 20 patients with SD in response to sorafenib treatment, only 4

$220(20 \%)$ had PR to lenvatinib treatment, and most patients (the other 16 patients, $80 \%$ ) remained

221 stable with lenvatinib treatment. Among 14 patients who had disease progression with sorafenib 
222 treatment, only 1 (7.1\%) had PR to lenvatinib treatment, and the other 13 patients showed poor

223 response to lenvatinib treatment. A comparison of treatment response between sorafenib and

224 lenvatinib is presented in Table 5.

225

226

Adverse events associated with lenvatinib

227

The AEs of lenvatinib treatment were informed to most patients. AEs with higher

228

frequencies (> 20\%) included hypertension, diarrhea, decreased appetite, fatigue, and palmar-

229

plantar erythrodysesthesia. Most AEs were grade 1-2; grade 3-4 toxicities were rare, including

230

hypertension $(12.5 \%)$, diarrhea $(2.5 \%)$, decreased appetite $(2.5 \%)$, and fatigue $(2.5 \%)$. The

231 majority of the patients tolerated the side effects of lenvatinib treatment, and no patients had

232

treatment-related deaths. The profiles of these AEs are shown in Table 6.

233

234

\section{Discussion}

235

HCC is an extremely aggressive malignant cancer, and its treatment is extremely

236

challenging with advanced tumor status. For the past 10 years, sorafenib therapy has been the

237

only recommended first-line systemic treatment for unresectable HCC (Cheng et al. 2009; Llovet 
238 et al. 2008). Although several clinical trials were conducted with several compounds that were

239 designed to be superior or non-inferior to sorafenib, all of them failed, and none were approved

240 (Cainap et al. 2015; Cheng et al. 2013; Johnson et al. 2013). Recently, the REFLECT trial

241 showed that lenvatinib therapy is non-inferior to sorafenib in terms of OS (Kudo et al. 2018).

242 Subsequently, lenvatinib was used as the first-line treatment for unresectable HCC in some

243 countries, especially in Japan, and several real-world studies demonstrated that lenvatinib

244 treatment yields good response and has safety (Hiraoka et al. 2019a; Hiraoka et al. 2019b; Obi et

245 al. 2019). However, the clinical impact of lenvatinib treatment in patients who received sorafenib

246 treatment remains limited. In our study, the PFS and OS were 3.3 and 9.8 months, respectively,

247 in patients who received lenvatinib after sorafenib; the outcome was similar to that of other

248 second-line treatments for HCC in the previous phase III trials (Bruix et al. 2017; Zhu et al.

249 2019). In addition, AFP $>400 \mathrm{ng} / \mathrm{mL}$ was noted as an independent poor prognostic factor of PFS

250 and OS in univariate and multivariate analyses.

251 In the REFLECT trial, patients with Child-Pugh classification B or main PVT were

252 excluded; thus, the efficacy and safety of lenvatinib for these patients are still unclear. However,

253 some patients who had Child-Pugh classification B or main PVT are still treated with lenvatinib 
254 in real-world practice. In our study, we enrolled 9 (22.5\%) patients with Child-Pugh

255 classification B and 11 (27.5\%) patients with main PVT who received lenvatinib therapy as

256 systemic treatment. The median PFS and OS were worse in patients with Child-Pugh

257 classification B or main PVT than in those in patients with Child-Pugh classification A or non-

258 main PVT, although the difference was not significant. This effect was also observed in 19

$259(47.5 \%)$ patients with macrovascular invasion. In contrast, patients who underwent hepatectomy

260 before lenvatinib treatment had better PFS than those who did not, although there was no

261 significant difference. However, the benefit of OS was marginally significant in the hepatectomy

262 group than in the non-hepatectomy group. This could be due to the small size of the patient

263 population in our study.

264 Sorafenib and lenvatinib have been used in first-line systemic treatment for unresectable

$265 \mathrm{HCC}$, and patients who received lenvatinib as second-line or later line treatment were not

266 enrolled in the REFLECT trial. However, some patients may have received other therapeutic

267 modalities before lenvatinib treatment in clinical practice, such as sorafenib therapy, hepatic

268 arterial infusion chemotherapy, or immunotherapy; thus, lenvatinib therapy is used as second-

269 line or later line treatment rather than first-line treatment. In our study, the median PFS and OS 
270 were similar among the second-line, third-line, and fourth-line and later line treatments, although

271 the OS of the second-line treatment was mildly worse than that of the third-line and later line

272 treatments, indicating the efficacy of lenvatinib as second-line and later line treatments for

273 unresectable HCC.

274 The response rate is also an important issue in HCC treatment. Our study showed that the

275 median PFS was better in responders than in patients with SD or PD, but the 1-year OS rate was

276 equal between patients with PR or SD. In addition, the response to previous sorafenib could

277 predict the response of subsequent lenvatinib. In our study, all patients who responded to

278 sorafenib had treatment response to lenvatinib; most patients $(80 \%)$ with SD as a response to

279 sorafenib treatment remained in a stable condition with lenvatinib treatment. Patients without

response to sorafenib were still not found to be responsive to lenvatinib $(92.9 \%)$. The correlation

281 between treatment response to sorafenib and lenvatinib is extremely helpful in the treatment of

282 HCC in clinical practice.

283 Our study had several limitations. First, the study had a retrospective design, with a small

284 sample size, and all patients were treated at a single institution. Second, the number of female

285 patients in our study was limited; therefore, a bias of patient sex might have existed. Third, the 
286 follow-up period may not be adequately long, and some survival benefit may not be significant.

287 However, to the best of our knowledge, this is the first study designed to investigate the clinical

288 impact of lenvatinib as second-line and later line treatment in patients with unresectable HCC

289 who received sorafenib. Further studies with a larger population or a randomized controlled trial

290 are warranted to validate the findings of this study.

291

292

293

294

295 Conclusions

Our study confirms the efficacy and safety of lenvatinib for use as second-line and later line 


\section{Competing interests}

312 The authors declare that they have no competing interests.

313

\section{Authors' Contributions}

315 YHC conceived and designed the experiments. YYC wrote the main manuscript text. CCW

316 and YWL performed the experiments. WFL prepared the tables and figures. All authors

317 reviewed the manuscript. 


\section{Funding}

320 The authors received no financial support for this research.

321

322

323

324

325

326

327

328

329 Figure Legends

330 Figure 1. Kaplan-Meier survival curves of progression-free survival (PFS) and overall survival

331 (OS) among patients with unresectable hepatocellular carcinoma who received lenvatinib treatment as second-line or later line treatment. (A) PFS and (B) OS. 
334 Figure 2. Comparison of survival curves of progression-free survival (PFS) and overall survival

335 (OS) between patients with unresectable hepatocellular carcinoma who had AFP $>400 \mathrm{ng} / \mathrm{mL}$ or

336 AFP $<400 \mathrm{ng} / \mathrm{mL}$. (A) PFS and (B) OS.

337

338

339

340

341

342

343

344

345 Reference

346 Common Terminology Criteria for Adverse Events v4.0 (CTCAE)

347 May 28, 2009 NIH Publication No. 03-5410

348 https://ctep.cancer.gov/protocolDevelopment/electronic_applications/ctc.htm\#ctc_50.

349 National Department of Health, Republic of China. Cancer Registry Annual Report 2015. 
350 Bertuccio P, Turati F, Carioli G, Rodriguez T, La Vecchia C, Malvezzi M, and Negri E. 2017. Hepatology 67:302-309. 10.1016/j.jhep.2017.03.011

Bruix J, Qin S, Merle P, Granito A, Huang YH, Bodoky G, Pracht M, Yokosuka O, Rosmorduc placebo-controlled, phase 3 trial. Lancet 389:56-66. 10.1016/S0140-6736(16)32453-9 10.1053/j.gastro.2015.12.041

Cabibbo G, Maida M, Genco C, Parisi P, Peralta M, Antonucci M, Brancatelli G, Camma C, A retrospective cohort study. World Journal of Hepatology 4:256-261. 
366 Cainap C, Qin S, Huang WT, Chung IJ, Pan H, Cheng Y, Kudo M, Kang YK, Chen PJ, Toh HC, results of a randomized phase III trial. Journal of Clinical Oncology 33:172-179. Z. 2009. Efficacy and safety of sorafenib in patients in the Asia-Pacific region with advanced hepatocellular carcinoma: a phase III randomised, double-blind, placebocontrolled trial. Lancet Oncology 10:25-34. 10.1016/S1470-2045(08)70285-7 randomized phase III trial. Journal of Clinical Oncology 31:4067-4075. 
381 Hiraoka A, Kumada T, Atsukawa M, Hirooka M, Tsuji K, Ishikawa T, Takaguchi K, Kariyama

Tsutsui A, Nagano T, Itokawa N, Hayama K, Imai M, Joko K, Koizumi Y, Hiasa Y,

Michitaka K, Kudo M, and Real-life Practice Experts for Hcc Study Group HCCG.

385

2019a. Prognostic factor of lenvatinib for unresectable hepatocellular carcinoma in realworld conditions-Multicenter analysis. Cancer Med 8:3719-3728. 10.1002/cam4.2241

Hiraoka A, Kumada T, Kariyama K, Takaguchi K, Atsukawa M, Itobayashi E, Tsuji K, Tajiri K,

Hirooka M, Shimada N, Shibata H, Ishikawa T, Ochi H, Tada T, Toyoda H, Nouso K,

Tsutsui A, Itokawa N, Imai M, Joko K, Hiasa Y, Michitaka K, and Real-life Practice unresectable hepatocellular carcinoma in real-world conditions: Multicenter analysis.

Hiraoka A, Kumada T, Kariyama K, Takaguchi K, Itobayashi E, Shimada N, Tajiri K, Tsuji K, 2019c. Therapeutic potential of lenvatinib for unresectable hepatocellular carcinoma in 
clinical practice: Multicenter analysis. Hepatology Research 49:111-117.

Johnson PJ, Qin S, Park JW, Poon RT, Raoul JL, Philip PA, Hsu CH, Hu TH, Heo J, Xu J, Lu L, advanced hepatocellular carcinoma: results from the randomized phase III BRISK-FL 
412 Llovet JM, Bru C, and Bruix J. 1999. Prognosis of hepatocellular carcinoma: the BCLC staging

413 classification. Seminars in Liver Disease 19:329-338. 10.1055/s-2007-1007122

414 Llovet JM, Ricci S, Mazzaferro V, Hilgard P, Gane E, Blanc JF, de Oliveira AC, Santoro A,

415 Raoul JL, Forner A, Schwartz M, Porta C, Zeuzem S, Bolondi L, Greten TF, Galle PR,

416 Seitz JF, Borbath I, Haussinger D, Giannaris T, Shan M, Moscovici M, Voliotis D, Bruix

417 J, and Group SIS. 2008. Sorafenib in advanced hepatocellular carcinoma. New England

$418 \quad$ Journal of Medicine 359:378-390. 10.1056/NEJMoa0708857

419 Marrero JA, Kulik LM, Sirlin CB, Zhu AX, Finn RS, Abecassis MM, Roberts LR, and

420 Heimbach JK. 2018. Diagnosis, Staging, and Management of Hepatocellular Carcinoma:

4212018 Practice Guidance by the American Association for the Study of Liver Diseases.

422 Hepatology 68:723-750. 10.1002/hep.29913

423 Obi S, Sato T, Sato S, Kanda M, Tokudome Y, Kojima Y, Suzuki Y, Hosoda K, Kawai T,

424 Kondo Y, Isomura Y, Ohyama H, Nakagomi K, Ashizawa H, Miura Y, Amano H,

425 Mochizuki H, and Omata M. 2019. The efficacy and safety of lenvatinib for advanced

426 hepatocellular carcinoma in a real-world setting. Hepatology International 13:199-204.

427

10.1007/s12072-019-09929-4 
428 Omata M, Cheng AL, Kokudo N, Kudo M, Lee JM, Jia J, Tateishi R, Han KH, Chawla YK, Shiina S, Jafri W, Payawal DA, Ohki T, Ogasawara S, Chen PJ, Lesmana CRA, Lesmana International 11:317-370. 10.1007/s12072-017-9799-9

Schlumberger M, Tahara M, Wirth LJ, Robinson B, Brose MS, Elisei R, Habra MA, Newbold K, 10.1056/NEJMoa1406470 Hiriart JB, Okusaka T, Morimoto M, Hsu Y, Abada PB, Kudo M, and investigators R-s. 2019. Ramucirumab after sorafenib in patients with advanced hepatocellular carcinoma and increased alpha-fetoprotein concentrations (REACH-2): a randomised, double-blind, 
443 placebo-controlled, phase 3 trial. Lancet Oncology 20:282-296. 10.1016/S1470- 


\section{Table $\mathbf{1}$ (on next page)}

Characteristics of 40 patients with unresectable hepatocellular carcinoma who received lenvatinib after failure of sorafenib 
Table 1. Characteristics of 40 patients with unresectable hepatocellular carcinoma who received lenvatinib after failure of sorafenib

Characteristics

Age (median)

Body weight

Liver cirrhosis

ECOG performance status

0

1

Sex

Male

Female

Child-Pugh classification

A

B

BCLC classification

B

C

Viral hepatitis status

Hepatitis B

Hepatitis C

No

Main portal vein thrombosis

Yes

No

Macrovascular invasion

Yes

No

Hepatectomy before lenvatinib

Yes

No

Extrahepatic spread

Yes

No

AFP $>400$

Yes

No
58 years (34-78)

$66.2 \mathrm{~kg}(44.8-109.5)$

$40(100 \%)$

$6(15.0 \%)$

$34(85.0 \%)$

$35(87.5 \%)$

$5(12.5 \%)$

$31(77.5 \%)$

$9(22.5 \%)$

$3(7.5 \%)$

37 (92.5\%)

29 (72.5\%)

$7(17.5 \%)$

$4(10.0 \%)$

$11(27.5 \%)$

29 (72.5\%)

19 (47.5\%)

21 (52.5\%)

$24(60.0 \%)$

$16(40.0 \%)$

$32(80.0 \%)$

$8(20.0 \%)$

17 (42.5\%)

$23(57.5 \%)$ 
ECOG: Eastern Cooperative Oncology Group; BCLC: Barcelona-Clinic Liver Cancer 


\section{Table 2 (on next page)}

Univariate and multivariate analyses of PFS in 40 patients with unresectable hepatocellular carcinoma who received lenvatinib after failure of sorafenib 
Table 2. Univariate and multivariate analyses of PFS in 40 patients with unresectable hepatocellular carcinoma who received lenvatinib after failure of sorafenib

\begin{tabular}{|c|c|c|c|c|c|}
\hline \multirow{2}{*}{ Characteristics } & \multirow{2}{*}{$\begin{array}{l}\text { No. of } \\
\text { patients }\end{array}$} & \multicolumn{2}{|l|}{ Univariate } & \multicolumn{2}{|c|}{ Multivariate } \\
\hline & & Median PFS (months) & $P$ value & HR $(95 \%$ CI $)$ & $P$ value \\
\hline \multicolumn{6}{|l|}{ Age } \\
\hline$<60$ years & $22(55.0 \%)$ & 3.2 & 0.40 & & \\
\hline$\geq 60$ years & $18(45.0 \%)$ & 3.4 & & & \\
\hline \multicolumn{6}{|c|}{ ECOG performance status } \\
\hline 0 & $6(15.0 \%)$ & 2.7 & 0.70 & & \\
\hline 1 & $34(85.0 \%)$ & 3.3 & & & \\
\hline \multicolumn{6}{|l|}{ Sex } \\
\hline Male & $35(87.5 \%)$ & 3.5 & 0.22 & & \\
\hline Female & $5(12.5 \%)$ & 2.9 & & & \\
\hline \multicolumn{6}{|c|}{ Child-Pugh classification } \\
\hline A & $31(77.5 \%)$ & 3.5 & 0.31 & & \\
\hline B & $9(22.5 \%)$ & 2.9 & & & \\
\hline \multicolumn{6}{|c|}{ BCLC staging classification } \\
\hline B & $3(7.5 \%)$ & 8.2 & 0.22 & & \\
\hline $\mathrm{C}$ & $37(92.5 \%)$ & 3.3 & & & \\
\hline \multicolumn{6}{|l|}{ Hepatitis B } \\
\hline Yes & $29(72.5 \%)$ & 5.6 & 0.26 & & \\
\hline No & $11(27.5 \%)$ & 3.2 & & & \\
\hline \multicolumn{6}{|l|}{ Hepatitis C } \\
\hline Yes & $7(17.5 \%)$ & 3.3 & 0.30 & & \\
\hline No & $33(82.5 \%)$ & 6.7 & & & \\
\hline Main portal vein tl & & & & & \\
\hline
\end{tabular}




\begin{tabular}{|c|c|c|c|c|c|}
\hline Yes & $11(27.5 \%)$ & 3.1 & \multirow[t]{2}{*}{0.53} & & \\
\hline No & $29(72.5 \%)$ & 3.7 & & & \\
\hline \multicolumn{6}{|c|}{ crovascular invasion } \\
\hline Yes & $19(47.5 \%)$ & 2.9 & \multirow{2}{*}{0.99} & & \\
\hline No & $21(52.5 \%)$ & 3.5 & & & \\
\hline \multicolumn{6}{|c|}{ patectomy before lenvatinib treatment } \\
\hline Yes & $24(60.0 \%)$ & 3.4 & \multirow[t]{2}{*}{0.62} & & \\
\hline No & $16(40.0 \%)$ & 2.9 & & & \\
\hline \multicolumn{6}{|c|}{ rahepatic spread } \\
\hline Yes & $32(80.0 \%)$ & 3.1 & \multirow{2}{*}{\multicolumn{2}{|c|}{0.27}} & \\
\hline No & $8(20.0 \%)$ & 4.6 & & & \\
\hline \multicolumn{6}{|c|}{ P level $>400$} \\
\hline Yes & $17(42.5 \%)$ & 2.7 & \multicolumn{3}{|l|}{$0.020^{*}$} \\
\hline No & $23(57.5 \%)$ & 4.2 & & $0.46(0.23-0.90)$ & $0.024 *$ \\
\hline
\end{tabular}

HR: hazard ratio; CI: confidence interval; ECOG: Eastern Cooperative Oncology Group; PFS: progression-free survival; BCLC:

Barcelona Clinic Liver Cancer. *Statistically significant. 


\section{Table 3(on next page)}

Univariate and multivariate analyses of OS in 40 patients with unresectable hepatocellular carcinoma who received lenvatinib after failure of sorafenib 
Table 3. Univariate and multivariate analyses of OS in 40 patients with unresectable hepatocellular carcinoma who received lenvatinib after failure of sorafenib

\begin{tabular}{|c|c|c|c|c|c|}
\hline \multirow{2}{*}{ Characteristics } & \multirow{2}{*}{$\begin{array}{l}\text { No. of } \\
\text { patients }\end{array}$} & \multicolumn{2}{|l|}{ Univariate } & \multicolumn{2}{|c|}{ Multivariate } \\
\hline & & Median OS (months) & $P$ value & HR $(95 \% \mathrm{CI})$ & $P$ value \\
\hline \multicolumn{6}{|l|}{ Age } \\
\hline$<60$ years & $22(55.0 \%)$ & 12.0 & 0.93 & & \\
\hline$\geq 60$ years & $18(45.0 \%)$ & 9.8 & & & \\
\hline \multicolumn{6}{|c|}{ ECOG performance status } \\
\hline 0 & $6(15.0 \%)$ & 16.4 & 0.34 & & \\
\hline 1 & $34(85.0 \%)$ & 8.4 & & & \\
\hline \multicolumn{6}{|l|}{ Sex } \\
\hline Male & $35(87.5 \%)$ & 11.5 & 0.48 & & \\
\hline Female & $5(12.5 \%)$ & 3.5 & & & \\
\hline \multicolumn{6}{|c|}{ Child-Pugh classification } \\
\hline A & $31(77.5 \%)$ & 11.5 & 0.99 & & \\
\hline B & $9(22.5 \%)$ & 8.1 & & & \\
\hline \multicolumn{6}{|c|}{ BCLC staging classification } \\
\hline $\mathrm{B}$ & $3(7.5 \%)$ & 16.4 & 0.74 & & \\
\hline $\mathrm{C}$ & $37(92.5 \%)$ & 9.8 & & & \\
\hline \multicolumn{6}{|l|}{ Hepatitis B } \\
\hline Yes & $29(72.5 \%)$ & NR & 0.23 & & \\
\hline No & $11(27.5 \%)$ & 8.4 & & & \\
\hline \multicolumn{6}{|l|}{ Hepatitis C } \\
\hline Yes & $7(17.5 \%)$ & 9.8 & 0.16 & & \\
\hline No & $33(82.5 \%)$ & NR & & & \\
\hline Main portal vein th & & & & & \\
\hline
\end{tabular}




\begin{tabular}{|c|c|c|c|c|c|}
\hline Yes & $11(27.5 \%)$ & 12.0 & \multirow[t]{2}{*}{0.53} & & \\
\hline No & $29(72.5 \%)$ & 8.1 & & & \\
\hline \multicolumn{6}{|c|}{ crovascular invasion } \\
\hline Yes & $19(47.5 \%)$ & 12.0 & \multirow[t]{2}{*}{0.13} & & \\
\hline No & $21(52.5 \%)$ & 6.2 & & & \\
\hline \multicolumn{6}{|c|}{ patectomy before lenvatinib treatment } \\
\hline Yes & $24(60.0 \%)$ & 13.1 & \multirow[t]{2}{*}{0.09} & & \\
\hline No & $16(40.0 \%)$ & 6.7 & & & \\
\hline \multicolumn{6}{|c|}{ trahepatic spread } \\
\hline Yes & $32(80.0 \%)$ & 9.8 & \multirow[t]{2}{*}{0.20} & & \\
\hline No & $8(20.0 \%)$ & NR & & & \\
\hline \multicolumn{6}{|c|}{ P leel $>400$} \\
\hline Yes & $17(42.5 \%)$ & 6.1 & \multirow[t]{2}{*}{$<0.001 *$} & & \\
\hline No & $23(57.5 \%)$ & NR & & $0.19(0.08-0.46)$ & $<0.001 *$ \\
\hline
\end{tabular}

HR: hazard ratio; CI: confidence interval; ECOG: Eastern Cooperative Oncology Group; OS: overall survival; BCLC: Barcelona Clinic Liver Cancer; NR: not reach.*Statistically significant. 


\section{Table 4(on next page)}

Survival analyses of the 40 patients with HCC who received lenvatinib after failure of sorafenib 
Table 4. Survival analyses of the $\mathbf{4 0}$ patients with $\mathrm{HCC}$ who received lenvatinib after failure of sorafenib

\begin{tabular}{|c|c|c|c|c|c|}
\hline Response to lenvatinib & $\begin{array}{c}\text { Number of } \\
\text { patients }\end{array}$ & $\begin{array}{c}\text { 6-month PFS rate } \\
(\%)\end{array}$ & $\mathrm{P}$ value & $\begin{array}{c}\text { 1-year OS rate } \\
(\%)\end{array}$ & $\begin{array}{c}\mathrm{P} \\
\text { value }\end{array}$ \\
\hline Complete response & $1(2.5 \%)$ & & \multirow{4}{*}{$0.046^{*}$} & & \multirow{4}{*}{0.20} \\
\hline Partial response & $10(25.0 \%)$ & 45.7 & & 53.3 & \\
\hline Stable disease & $16(40.0 \%)$ & 18.8 & & 55.6 & \\
\hline Progressive disease & $13(32.5 \%)$ & 0 & & 8.5 & \\
\hline Lenvatinib treatment lines & $\begin{array}{c}\text { Number of } \\
\text { patients }\end{array}$ & PFS (months) & $P$ value & OS (months) & $\begin{array}{c}\mathrm{P} \\
\text { value }\end{array}$ \\
\hline Second line & $20(50.0 \%)$ & 3.1 & \multirow{3}{*}{0.38} & 8.1 & \multirow{3}{*}{0.98} \\
\hline Third line & $10(25.0 \%)$ & 3.7 & & 11.5 & \\
\hline $\begin{array}{l}\text { Fourth line and later } \\
\text { lines }\end{array}$ & $10(25.0 \%)$ & 3.1 & & 12.0 & \\
\hline
\end{tabular}

HCC: hepatocellular carcinoma; PFS: progression-free survival; OS: overall survival; *Statistically significant. 
Table 5 (on next page)

Comparison of treatment response to sorafenib and lenvatinib in the 40 patients with $\mathrm{HCC}$ 
Table 5. Comparison of treatment response to sorafenib and lenvatinib in the 40 patients with $\mathrm{HCC}$

\begin{tabular}{lcccc}
\hline \multicolumn{1}{c}{ Response to lenvatinib } & CR and PR & SD & PD & P value \\
\cline { 1 - 2 } Response to previous sorafenib & & & & \\
\cline { 1 - 1 }$(\mathrm{N}=6)$ & $6(100 \%)$ & $0(0 \%)$ & $0(0 \%)$ & \\
SD $(\mathrm{N}=20)$ & $4(20 \%)$ & $16(80 \%)$ & $0(0 \%)$ & $\mathrm{P}<0.001^{*}$ \\
$\mathrm{PD}(\mathrm{N}=14)$ & $1(7.1 \%)$ & $0(0 \%)$ & $13(92.9 \%)$ & \\
\hline
\end{tabular}

HCC: hepatocellular carcinoma; CR: complete response; PR: partial response; SD: stable disease; PD: progressive disease; *Statistically significant. 
Table 6(on next page)

Treatment-related adverse events of the 40 patients with unresectable hepatocellular carcinoma receiving lenvatinib after failure of sorafenib 
Table 6. Treatment-related adverse events of the 40 patients with unresectable hepatocellular carcinoma receiving lenvatinib after failure of sorafenib

\begin{tabular}{|c|c|c|c|c|c|c|c|c|}
\hline \multirow[b]{2}{*}{ Adverse event } & \multicolumn{2}{|c|}{ All patients $(\mathrm{N}=40)$} & \multicolumn{2}{|c|}{ Second line $(\mathrm{N}=20)$} & \multicolumn{2}{|c|}{ Third line $(\mathrm{N}=10)$} & \multicolumn{2}{|c|}{$\begin{array}{l}\text { Fourth and later line } \\
\qquad(\mathrm{N}=10)\end{array}$} \\
\hline & Any grades & Grade 3/4 & Any grades & Grade 3/4 & $\begin{array}{l}\text { Any } \\
\text { grades }\end{array}$ & Grade 3/4 & Any grades & Grade 3/4 \\
\hline Hypertension & $19(47.5 \%)$ & $5(12.5 \%)$ & $9(45.0 \%)$ & $3(15.0 \%)$ & $6(60.0 \%)$ & $1(10.0 \%)$ & $4(40.0 \%)$ & $1(10.0 \%)$ \\
\hline Diarrhea & $14(35.0 \%)$ & $1(2.5 \%)$ & $6(30.0 \%)$ & $0(0 \%)$ & $4(40.0 \%)$ & $1(10.0 \%)$ & $4(40.0 \%)$ & $0(0 \%)$ \\
\hline Decreased appetite & $9(22.5 \%)$ & $1(2.5 \%)$ & $4(20.0 \%)$ & $1(5.0 \%)$ & $3(30.0 \%)$ & $0(0 \%)$ & $2(20.0 \%)$ & $0(0 \%)$ \\
\hline Decreased body weight & $7(17.5 \%)$ & $0(0 \%)$ & $4(20.0 \%)$ & $0(0 \%)$ & $1(10.0 \%)$ & $0(0 \%)$ & $2(20.0 \%)$ & $0(0 \%)$ \\
\hline Fatigue & $11(27.5 \%)$ & $1(2.5 \%)$ & $7(35.0 \%)$ & $1(5.0 \%)$ & $2(20.0 \%)$ & $0(0 \%)$ & $2(20.0 \%)$ & $0(0 \%)$ \\
\hline $\begin{array}{l}\text { Palmar-plantar } \\
\text { erythrodysesthesia }\end{array}$ & $8(20.0 \%)$ & $0(0 \%)$ & $3(15.0 \%)$ & $0(0 \%)$ & $3(30.0 \%)$ & $0(0 \%)$ & $2(20.0 \%)$ & $0(0 \%)$ \\
\hline Nausea & $6(15.0 \%)$ & $0(0 \%)$ & $3(15.0 \%)$ & $0(0 \%)$ & $2(20.0 \%)$ & $0(0 \%)$ & $1(10.0 \%)$ & $0(0 \%)$ \\
\hline Vomiting & $2(5.0 \%)$ & $0(0 \%)$ & $1(5.0 \%)$ & $0(0 \%)$ & $1(10.0 \%)$ & $0(0 \%)$ & $0(0 \%)$ & $0(0 \%)$ \\
\hline Skin rash & $3(7.5 \%)$ & $0(0 \%)$ & $1(5.0 \%)$ & $0(0 \%)$ & $1(10.0 \%)$ & $0(0 \%)$ & $1(10.0 \%)$ & $0(0 \%)$ \\
\hline
\end{tabular}


Figure 1

Survival outcome of lenvatinib as second-line or later line treatment.

Kaplan-Meier survival curves of progression-free survival (PFS) and overall survival (OS) among patients with unresectable hepatocellular carcinoma who received lenvatinib treatment as second-line or later line treatment. (A) PFS and (B) OS.

(A)

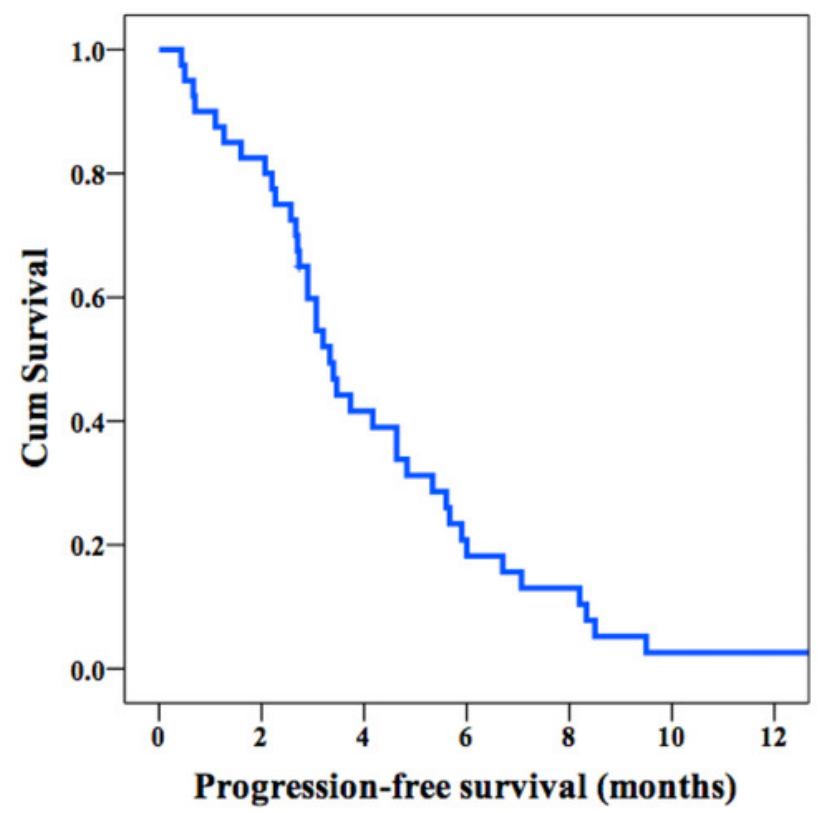

(B)

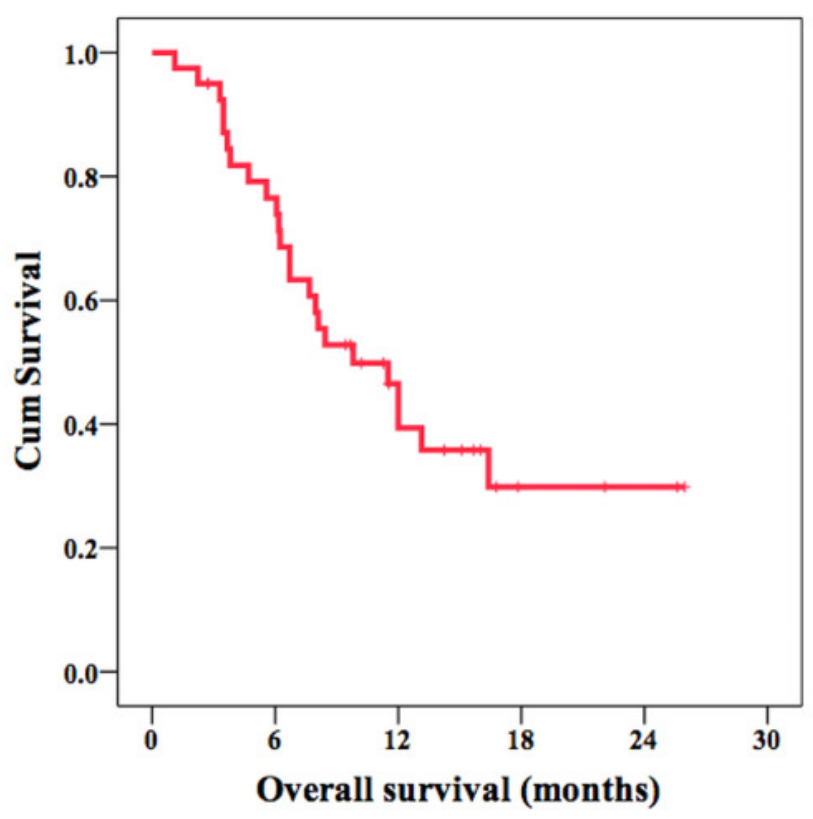


Figure 2

Correlation of AFP level and survival outcome.

Comparison of survival curves of progression-free survival (PFS) and overall survival (OS) between patients with unresectable hepatocellular carcinoma who had AFP $>400 \mathrm{ng} / \mathrm{mL}$ or AFP $<400 \mathrm{ng} / \mathrm{mL}$. (A) PFS and (B) OS.
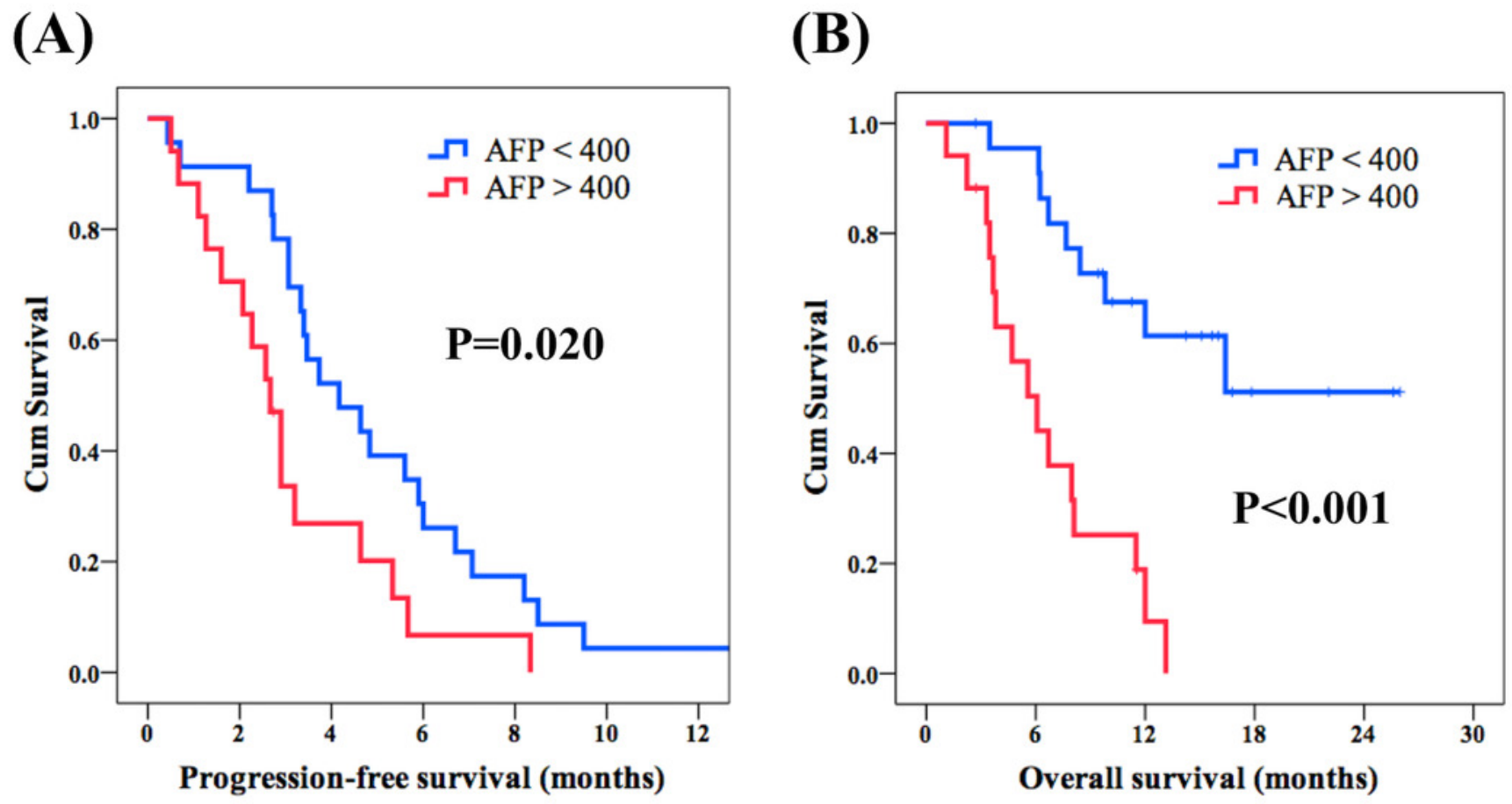